\title{
Differences in adolescent effective contraception use between population densities
}

\author{
Tess M Crouss ${ }^{1 *}$, Ruofan Yao $^{2}$ and Ryan Brannon ${ }^{1}$ \\ ${ }^{1}$ Department of Obstetrics and Gynecology, Drexel University College of Medicine, Philadelphia, USA \\ ${ }^{2}$ Department of Obstetrics and Gynecology, University of Maryland School of Medicine, Baltimore, USA
}

\begin{abstract}
Objective: Studies addressing effective contraceptive use by population density are lacking. We hypothesize that contraception counseling and effective contraception use vary by population density.

Study design: This is a cross-sectional study using the 2006-2010 National Survey of Family Growth data, including female subjects ages 15 to 19 . The primary exposure was population density, defined as Principal city of a Metropolitan Statistical Area (MSA) or "city", Other MSA or "non-city urban", and Not MSA or "rural." The primary outcome was effective contraception use and the secondary outcome was contraceptive counseling exposure. Multivariate analysis using logistic regression was performed to estimate the association between population density and effective contraception use, as well as the likelihood of receiving contraceptive counseling.
\end{abstract}

Results: 2284 subjects were studied. Compared to non-city urban dwellers, city adolescents had similar effective contraception use (aOR: 0.99, [0.79, 1.24]), whereas rural adolescents had significantly higher use (aOR: $1.79,[1.35,2.36])$. Among sexually active respondents who were not using contraception, the rate of contraception counseling in non-city urban adolescents was 66.7\%. In comparison, the city dwellers had higher rate of counseling (79.1\%, aOR: 1.87; 95\% CI: [1.09, 3.22]). Similarly, rural adolescents also had higher rate of counseling (81.5\%, aOR: 2.37 ; $95 \%$ CI: [1.08, 5.19]).

Conclusion: Rural residents were more likely to use effective contraception methods than their city and non-city urban counterparts. However, higher rates of contraception counseling among sexually active adolescents not using contraception in city and rural densities could suggest ineffective counseling in these groups.

\section{Introduction}

Undesired pregnancy is a particular problem in the adolescent population. Close to $90 \%$ of the pregnancies in this population are unintended and account for significant economic and health burdens [1]. An estimated $\$ 11$ billion were spent in 2006 on unintended pregnancy alone [2]. The maternal risks of unintended pregnancy include increased incidence of depression and physical violence [3-7]. Teens experiencing unintended pregnancies have a lower likelihood of high school graduation and lower income achievement $[8,9]$. The fetal risks include less exposure to breast-feeding, delayed prenatal care initiation, increased incidence of birth defects, lower birth weight and lower overall education attainment [3-9]. These negative effects fuel a self-fulfilling cycle from generation to generation that inevitably leads to future unintended pregnancy.

The National Survey of Family Growth (NSFG) 2010 data reveals that while $78 \%$ of females and $85 \%$ of males used contraception during first episode of intercourse, their most common method was condoms, a highly unreliable method of contraception [10]. Current recommendations from the American College of Obstetricians and Gynecologists (ACOG) and the American Academy of Pediatrics encourage the use of long acting reversible contraceptives (LARCs) in sexually active adolescents [11-13]. Despite this, a mere 6\% of highschool students used non-pill hormonal methods of contraception at first intercourse [10].

Counseling the adolescent population is especially challenging. The population have vast reproductive variability and sexual behaviors are highly influenced by the cultural norms established by their peer groups [14]. Disparities in adolescent pregnancy rates have several contributing factors, including differences in cultural or religious preferences, access to quality family planning services and contraceptive counseling, and insurance barriers. As demonstrated by the CHOICE project, if adolescents are provided with quality contraception counseling and access to family planning services, they will readily use highly effective methods of contraception like LARC, and will have significantly lower pregnancy rates than their counterparts [15]. Despite this, studies show that contraception counseling is not yet comprehensive and varies based on several factors including the type of clinic administering care [16]. We therefore would expect regional variations in contraception counseling effectiveness and contraception use. Identifying regional deficiencies will allow for a more targeted distribution of family planning efforts to achieve an eventual goal of reducing unintended adolescent pregnancy rates.

We wanted to explore whether contraception counseling and effective contraception use varies by population density. Therefore, we designed a study using the 2006-2010 NSFG database to determine

${ }^{*}$ Correspondence to: Tess Crouss, Department of Obstetrics and Gynecology, Drexel University College of Medicine, 245 N. 15th Street, MS 495, Philadelphia, PA 19102, USA, Tel: 207-751-9964; E-mail: crousst@gmail.com

Key words: contraception, adolescent, population density, unintended pregnancy

Received: April 09, 2018; Accepted: May 07, 2018; Published: May 10, 2018 
if there are differences in effective contraceptive use and unintended pregnancy rates among adolescents living in city, non-city urban and rural settings.

\section{Methods}

We performed a cross-sectional study using the NSFG 2006-2010 dataset. This database is constructed by the National Center for Health Statistics, which surveys a nationally representative sample of the US to collect data on reproductive health. The data was collected using inperson questions about reproductive health topics. Sensitive questions were offered to the participants via laptop response, also by an inperson encounter. The participants included over 12,000 females aged 15-44 nationwide $[10,17]$. For this analysis, we included all females between the age of 15 and 19, cumulating to a total of 2284 subjects. We excluded males and those outside of this age range.

The primary exposure of interest was the population density at each subject's registered address. The NSFG database used 2010 census population counts and definitions established by the U.S. Office of Management and Budget to categorize subjects into one of three population types based on density: Principal city of a Metropolitan Statistical Area (MSA), which we will refer to as "city", Other MSA or "non-city urban", and Not MSA or "rural." Loosely defined, an MSA is a county with at least 50,000 people with adjacent counties that are integrated via commuting ties $[10,18]$. A principal city is "the largest incorporated place with a population of at least 10,000 in a core based statistical area" $[10,18]$.

Contraception use was broken into the following categories: none, combined hormonal contraceptive methods (pill, patch, ring), intermediate acting contraceptives (depot medroxyprogesterone acetate), LARCs (progesterone implant, intrauterine devices), barrier methods (condoms and diaphragms), sterilization, pregnancy, and other (withdrawal, spermicidal agents, rhythm method). The primary outcome of interest was effective methods of contraception, which we defined as any of the following: combined hormonal methods, intermediate acting methods, LARCs and barrier methods (excluding sterilization or pregnancy). The secondary outcome of interest was contraception counseling (whether the subject reports receiving counseling or information about birth control from a medical care provider in the last 12 months).

Demographic information was compared between different population densities using the appropriate univariate statistics. Chi squared test was used for categorical variables, analysis of variance (ANOVA) was used for continuous variables. P value of $<0.05$ was deemed statistically significant. Multivariate analysis using logistic regression was performed to estimate the association of population density types on effective contraception use. Additionally, the association between population density and contraception counseling in sexually active subjects who are not using contraception was evaluated. The analyses were adjusted for age, race, prior pregnancy and history of undesired pregnancy. All analyses were performed using Stata 12 (College Station, TX). This study was approved by Drexel University's Institutional Review Board (IRB ID:1505003683).

\section{Results}

There were 2284 females aged 15-19 included for analysis, with a mean age of 17. Significant racial differences were present within the population density strata, with a greater proportion of black residents in city populations $(34.8 \%)$, compared to non-city urban $(13.2 \%)$ and rural areas $(14 \%),(\mathrm{p}<0.001)$. The city population comprised the highest percentage of adolescents who had ever been pregnant (16.4\%), compared to the rural (15.4\%) and non-city urban (9.6\%) populations, as well as the highest percentage of those who had been pregnant greater than or equal to 2 times (5.4\%), compared to rural $(4.2 \%)$ and non-city urban adolescents $(2.5 \%),(\mathrm{p}<0.001)$. There was no significant difference in percentages of undesired pregnancies across populations (city at $23.4 \%$, non-city urban at $16 \%$, rural at $16.4 \%, \mathrm{p}=0.63$ ), (Table 1 ).

Contraception use was different between population density strata, with a higher proportion of rural adolescents $(46.5 \%)$ using a current method of contraception, compared to city (36.6\%) and non-city urban $(32.5 \%)$ adolescent females, $(\mathrm{p}<0.001)$. Combined contraceptive methods were the most common across all populations, and LARC use was low. Using non-city urban adolescents as a reference (27.8\%), rural adolescents were more likely to use effective contraception methods (40.5\%, aOR: $1.79 ; 95 \%$ CI: $[1.35,2.36])$, whereas city adolescents has similar rates of use (29.9\%, aOR: 0.99; 95\% CI: [0.79, 1.24]). (Table 2).

Among sexually active respondents who are not using contraception (Table 3), the city population comprised the highest percentage of adolescents who had ever been pregnant (18.3\%), compared to the noncity urban (16.7\%) and rural (9.3\%) populations, as well as the highest percentage of those who had been pregnant greater than or equal to 2 times (6.5\%), compared to non-city urban $(2.2 \%)$ and rural adolescents (1.9\%), $(\mathrm{p}=0.184)$. Within this smaller cohort, significant differences existed in exposure to contraception counseling within the population density strata. The percentage of contraception counseling in non-city urban adolescents was $66.7 \%$. In comparison, the city dwellers had a significantly higher rate of counseling (79.1\%, aOR: 1.87; 95\% CI: [1.09, 3.22]). Similarly, rural adolescents also had higher rate of counseling (81.7\%, aOR: 2.37; 95\% CI: [1.08, 5.19]), (Table 4).

Table 1: Characteristics of female adolescents aged 15-19 in the NSFG by geographic distribution

\begin{tabular}{|l|c|c|c|c|}
\hline & City & $\begin{array}{c}\text { Non-city } \\
\text { urban }\end{array}$ & Rural & P-Value \\
\hline $\mathrm{N}(2284)$ & 884 & 1043 & 357 & \\
\hline$\%$ & 38.7 & 45.7 & 15.6 & \\
\hline Age (mean (SD)) & $17.2(1.4)$ & $17(1.4)$ & $17(1.4)$ & 0.009 \\
\hline Race & & & & $<0.001$ \\
\hline Black & $307.6(34.8)$ & $137.7(13.2)$ & $50(14)$ & \\
\hline White & $457(51.7)$ & $745.7(71.5)$ & $260(72.8)$ & \\
\hline Other & $119.3(13.5)$ & $158.5(15.2)$ & $47.1(13.2)$ & \\
\hline Total Pregnancies & & & & $<0.001$ \\
\hline none & $739(83.6)$ & $942.9(90.4)$ & $302(84.6)$ & \\
\hline 1 & $97.2(11)$ & $74.1(7.1)$ & $40(11.2)$ & \\
\hline$\geq 2$ & $47.7(5.4)$ & $26.1(2.5)$ & $15(4.2)$ & \\
\hline Livebirths & & & & 0.71 \\
\hline none & $311.2(35.2)$ & $427.6(41)$ & $136.4(38.2)$ & \\
\hline 1 & $457(51.7)$ & $531.9(51)$ & $188.1(52.7)$ & \\
\hline$\geq 2$ & $115.8(13.1)$ & $83.4(8)$ & $32.5(9.1)$ & \\
\hline Undesired & & & & 0.63 \\
\hline Pregnancies & & & & \\
\hline none & $677.1(76.6)$ & $876.1(84)$ & $298.5(83.6)$ & \\
\hline 1 & $164.4(18.6)$ & $135.6(13)$ & $45.3(12.7)$ & \\
\hline$\geq 2$ & $42.4(4.8)$ & $31.3(3)$ & $12.9(3.6)$ & \\
\hline & & & & \\
\hline
\end{tabular}

Table 2: Effective contraception use in adolescents by population density

\begin{tabular}{|l|c|c|c|}
\hline & N (698) (\%) & *aOR & \multicolumn{2}{|c|}{$\mathbf{9 5 \%}$ CI } \\
\hline Non-city urban (Reference) & $290(27.8)$ & \multicolumn{2}{|c|}{ Reference } \\
\hline City & $264(29.9)$ & 0.99 & $0.79-1.24$ \\
\hline Rural & $144(40.5)$ & 1.79 & $1.35-2.36$ \\
\hline
\end{tabular}

*adjusted for age, race, birth control education, prior pregnancy, unwanted pregnancy 
Table 3: Sexually Active Non-Users of Contraception

\begin{tabular}{|l|c|c|c|c|}
\hline & City & $\begin{array}{c}\text { Non-city } \\
\text { urban }\end{array}$ & Rural & P-Value \\
\hline N (345) & 153 & 138 & 54 & \\
\hline$\%$ & 44.4 & 40 & 15.7 & \\
\hline Mean age & 17.6 & 17.5 & 17 & 0.04 \\
\hline SD & 1.3 & 1.2 & 1.5 & \\
\hline Race & & & & $<0.001$ \\
\hline Black & 39.2 & 18.8 & 11.1 & \\
\hline White & 45.8 & 67.4 & 74.1 & \\
\hline Other & 15 & 13.8 & 14.8 & \\
\hline Total Pregnancies & & & & 0.184 \\
\hline none & 81.7 & 83.3 & 90.7 & \\
\hline 1 & 11.8 & 14.5 & 7.4 & \\
\hline$\geq 2$ & 6.5 & 2.2 & 1.9 & \\
\hline No Birth Control Education (\%) & 20.9 & 33.3 & 18.5 & 0.023 \\
\hline Birth Control Education after first & 43.3 & 33.3 & 11.1 & 0.367 \\
\hline intercourse & & & \\
\hline
\end{tabular}

Table 4: Contraception education among sexually active adolescents not using birth control

\begin{tabular}{|l|c|c|c|}
\hline & N (257) (\%) & *aOR & 95\% CI \\
\hline Non-city urban & $92(66.7)$ & \multicolumn{2}{|c|}{ reference } \\
\hline City & $121(79.1)$ & 1.87 & $1.09-3.22$ \\
\hline Rural & $44(81.7)$ & 2.37 & $1.08-5.19$ \\
\hline
\end{tabular}

\section{Discussion}

In this nationally representative sample of adolescent females, adolescents residing in rural populations were 1.79 times more likely to use effective contraception than adolescents of other populations. However, the pregnancy rate among rural adolescents was higher than non-city urban residents and comparable to city residents. Regarding the high-risk group of sexually active non-users of contraception, city and rural adolescents had higher exposure to birth control counseling, yet still failed to initiate using contraception compared to non-city urban adolescents. Additionally, these high-risk adolescents living in non-city urban environments who have significantly less exposure to contraception counseling, still have an overall lower pregnancy rate than their city cohorts.

These findings highlight important differences in contraception use and counseling between U.S. adolescent populations. It was particularly interesting to find that rural adolescents were more likely to use effective contraception methods, but did not have lower pregnancy rates. These findings suggest a dichotomy of the rural adolescent population, with a significant portion of adolescents using effective contraception and not getting pregnant, and the remaining portion becoming pregnant more frequently, whether due to desired fertility or undesired fertility without effective contraception use. There are differences in rural populations that are too granular to be captured in the NSFG categories, which leads to the clumping of those in very rural populations where women are known to have less access to family planning services with those in less rural areas and adequate access [19]. It is possible that those residing in less rural environments are receiving more effective contraception counseling and have a higher uptake of effective contraception, while those residing in extremely rural environments are not receiving counseling and getting pregnant more readily, although this was unable to be addressed in the study. Nonetheless, these variations are useful when imagining the implementation of targeted family planning services across the U.S.

Looking closer at the high-risk group of sexually active non-users of contraception emphasizes further population differences. Our finding that these high-risk rural and city adolescents had more exposure to contraception counseling than their non-city rural counterparts, yet still failed to initiate effective contraception, could suggest ineffective counseling in these populations. Contraception counseling encompasses a wide range of forms, and within this variation are inherent differences in quality. To meet criteria for contraceptive counseling in the NSFG dataset, the adolescents must have received information about birth control from a medical care provider in the last 12 months, which could range from receiving pamphlets that list methods, to more thorough counseling sessions with a gynecologist. Interestingly, our findings showed that exposure to contraception counseling in general was associated with more uptake of effective methods of birth control. However, there are clearly other variables affecting contraception use even when counseling is present, which stresses the importance of a combination of quality counseling and access to obtaining the methods.

As mentioned above, differences in access to obtaining contraception is likely part of the explanation for the variation in effective use of contraception and contraception counseling by population density. Contraception use is known to vary based on certain variables such as socioeconomic status and race [20]. There may also exist population disparities pertaining to access to family planning services. Women living in rural areas traditionally have less access to reproductive services compared to metropolitan women [19]. Therefore, while our findings that rural adolescents had a higher uptake of effective contraception seems contradictory, it possibly reflects the abovementioned wide variation in rural populations, from more desolate farm country to areas just outside suburban populations [19]. Regarding the quality of contraception counseling, this likely does differ between population densities, but again, it seems unlikely that rural settings with a traditionally lower quality reproductive health care would be receiving higher quality counseling [19].

This study has several strengths. Importantly is its large sample size of a nationally representative sample of the US adolescent population. Additionally, both in-person and computer-based methods of surveying were used to elicit respondents' answers in an attempt to minimize responder bias inherent when questioning people about sensitive health and reproductive topics. There were also limitations. Important to consider are the different variations of "rural" populations that exist, which were unable to be ascertained in this dataset. Additionally, given the study's strategy of obtaining information via self-report as opposed to objective measures, it has the potential of leading to ascertainment bias, specifically responder reporting bias. Where this was avoided to the best extent possible by ensuring confidentiality and providing both in-person and lap-top interviewing methods, subjects have a natural tendency to answer questions in a way that allows them to align with cultural beliefs [21]. This likely lead to a decrease in the overall reported number of undesired pregnancies and in increase in the overall reported contraception use, and therefore our results likely underestimate the true unintended pregnancy rate. And lastly, the analysis is based on a cross-sectional study rather than an experimental or prospective study, which naturally limits the conclusions we can draw.

\section{Conclusion}

In conclusion, there are differences in contraceptive use between rural and urban adolescents which warrant further study. This study stimulates several questions as it sheds light onto population differences in the U.S. adolescent population regarding contraception counseling and use, and pregnancy rates. Clear from the start is the need for better access to and increased uptake of effective methods of contraception. 
Also important is both the presence, and likely the quality of the counseling that accompanies. From this analysis, one could suggest that higher quality contraceptive counseling may be occurring in rural populations, considering their higher use of effective methods of contraception. More studies are needed to understand how to more effectively increase contraception knowledge across all populations, for an ever-changing group of adolescents.

\section{Implications}

Effective contraception use does vary between population densities in the adolescent population and does not necessarily correlate with lower unintended pregnancy rates.

\section{References}

1. Finer LB, Zolna MR (2014) Shifts in intended and unintended pregnancies in the United States, 2001-2008. Am J Public Health 104: S43-S48. [Crossref]

2. Sonfield A, Kost K, Gold RB, Finer LB (2011) The Public Costs of Births Resulting from Unintended Pregnancies: National and State-Level Estimates. Perspect Sex Reprod Health 43: 94-102. [Crossref]

3. Logan C, Holcombe E, Manlove J, Ryan S (2007) The consequences of unintended childbearing. Child Trends Natl Campaign to Prev Teen Pregnancy 28: 142-151.

4. Cheng D, Schwarz EB, Douglas E, Horon I (2009) Unintended pregnancy and associated maternal preconception, prenatal and postpartum behaviors. Contraception 79: 194-198. [Crossref]

5. Kost K, Landry DJ, Darroch JE (1998) Predicting Maternal Behaviors During Pregnancy: Does Intention Status Matter? Fam Plann Perspect 30: 79. [Crossref]

6. D'Angelo DV, Gilbert BC, Rochat RW, Santelli JS, Herold JM (2004) Differences between mistimed and unwanted pregnancies among women who have live births. Perspect Sex Reprod Health 36: 192-197. [Crossref]

7. D'Angelo D, Williams L, Morrow B, et al. (2007) Preconception and interconception health status of women who recently gave birth to a live-born infant--Pregnancy Risk Assessment Monitoring System (PRAMS), United States, 26 reporting areas, 2004. MMWR Surveill Summ 56: 1-35. [Crossref]
8. Hoffman SD, Kean TH (2006) By the Numbers Chairman Surgeon General Covenant House.

9. Hoffman SD, Maynard R (2008) Kids Having Kids: Economic Costs and Social Consequences of Teen Pregnancy. (2nd ed) Rowman \& Littlefield Publishers.

10. Lepkowski JM, Davis KE, Groves RM, Van Hoewyk JMWD (2010) The 2006-2010 National Survey of Family Growth: sample design and analysis of a continuous survey Vital Heal Stat 2: 1-36. [Crossref]

11. American Academy of Pediatrics (2014) AAP Updates Recommendations on Teen Pregnancy Prevention.

12. American College of Obstetricians and Gynecologists (2012) Adolescents and LongActing Reversible Contraception: Implants and Intrauterine Devices. Obs Gynecol 120 . 983-988.

13. American College of Obstetricians and Gynecologists (2009) Increasing Use of Contraceptive Implants and Intrauterine Devices to Reduce Unintended Pregnancy. Obs Gynecol 114: 1434-1438

14. Buhi ER, Goodson P (2007) Predictors of Adolescent Sexual Behavior and Intention: A Theory-Guided Systematic Review. J Adolesc Heal 40: 4-21. [Crossref]

15. Secura GM, Madden T, McNicholas C, Mullersman J, Buckel CM, et al. (2014) Provision of No-Cost, Long-Acting Contraception and Teenage Pregnancy. $N$ Engl $J$ Med 371: 1316-1323. [Crossref]

16. Liddon N, Steiner RJ, Martinez GM (2017) Provider Communication with Adolescent and Young Females during Sexual and Reproductive Health Visits: Findings from the 2011-2015 National Survey of Family Growth. Contraception 96: 22-28. [Crossref]

17. About the National Survey of Family Growth (2014) National Survey of Family Growth. Centers for Disease Control and Prevention.

18. SDMMSA (2010) Standards for Delineating Metropolitan and Micropolitan Statistica Areas Notice. Fed Regist 75: 1-8.

19. American College of Obstetricians and Gynecologists (2014) Health Disparities in Rural Women. Obs Gynecol 123: 384-388.

20. Dehlendorf C, Park SY, Emeremni C a, Comer D, Vincett K, Borrero S (2014) Racial/ ethnic disparities in contraceptive use: variation by age and women's reproductive experiences. Am J Obstet Gynecol 210: e1-e9. [Crossref]

21. Dodou D, De Winter JCF (2014) Social desirability is the same in offline, online, and paper surveys: A meta-analysis. Comput Human Behav 36: 487-495.

Copyright: $\odot 2018$ Crouss TM. This is an open-access article distributed under the terms of the Creative Commons Attribution License, which permits unrestricted use, distribution, and reproduction in any medium, provided the original author and source are credited. 